\title{
The zebrafish genome encodes the largest vertebrate repertoire of functional aquaporins with dual paralogy and substrate specificities similar to mammals
}

Angèle Tingaud-Sequeira ${ }^{1,2 \dagger}$, Magdalena Calusinska ${ }^{1,3 \dagger}$, Roderick N Finn ${ }^{4 \dagger}$, François Chauvigné ${ }^{\text {, Juanjo Lozano }}{ }^{5}$, Joan Cerdà $^{1^{*}}$

\begin{abstract}
Background: Aquaporins are integral membrane proteins that facilitate the transport of water and small solutes across cell membranes. These proteins are vital for maintaining water homeostasis in living organisms. In mammals, thirteen aquaporins (AQP0-12) have been characterized, but in lower vertebrates, such as fish, the diversity, structure and substrate specificity of these membrane channel proteins are largely unknown.

Results: The screening and isolation of transcripts from the zebrafish (Danio rerio) genome revealed eighteen sequences structurally related to the four subfamilies of tetrapod aquaporins, i.e., aquaporins (AQPO, -1 and -4 ), water and glycerol transporters or aquaglyceroporins (Glps; AQP3 and AQP7-10), a water and urea transporter (AQP8), and two unorthodox aquaporins (AQP11 and -12). Phylogenetic analyses of nucleotide and deduced amino acid sequences demonstrated dual paralogy between teleost and human aquaporins. Three of the duplicated zebrafish isoforms have unlinked loci, two have linked loci, while DrAqp8 was found in triplicate across two chromosomes. Genomic sequencing, structural analysis, and maximum likelihood reconstruction, further revealed the presence of a putative pseudogene that displays hybrid exons similar to tetrapod AQP5 and -1. Ectopic expression of the cloned transcripts in Xenopus laevis oocytes demonstrated that zebrafish aquaporins and Glps transport water or water, glycerol and urea, respectively, whereas DrAqp11b and -12 were not functional in oocytes. Contrary to humans and some rodents, intrachromosomal duplicates of zebrafish AQP8 were water and urea permeable, while the genomic duplicate only transported water. All aquaporin transcripts were expressed in adult tissues and found to have divergent expression patterns. In some tissues, however, redundant expression of transcripts encoding two duplicated paralogs seems to occur.

Conclusion: The zebrafish genome encodes the largest repertoire of functional vertebrate aquaporins with dual paralogy to human isoforms. Our data reveal an early and specific diversification of these integral membrane proteins at the root of the crown-clade of Teleostei. Despite the increase in gene copy number, zebrafish aquaporins mostly retain the substrate specificity characteristic of the tetrapod counterparts. Based upon the integration of phylogenetic, genomic and functional data we propose a new classification for the piscine aquaporin superfamily.
\end{abstract}

\footnotetext{
* Correspondence: joan.cerda@irta.es

† Contributed equally

'Laboratory of Institut de Recerca i Tecnologia Agroalimentàries (IRTA)-

Institut de Ciències del Mar, Consejo Superior de Investigaciones Científicas

(CSIC), 08003 Barcelona, Spain
}

\section{$\int$ Biomed Central}

(c) 2010 Tingaud-Sequeira et al; licensee BioMed Central Ltd. This is an Open Access article distributed under the terms of the Creative Commons Attribution License (http://creativecommons.org/licenses/by/2.0), which permits unrestricted use, distribution, and reproduction in any medium, provided the original work is properly cited. 


\section{Background}

Aquaporins constitute a superfamily of major intrinsic proteins (MIPs) that facilitate passive, yet remarkably efficient permeation of water molecules across cellular membranes $[1,2]$. Some aquaporins can also permeate non-ionic compounds, such as glycerol and urea, and are termed glycerol facilitators (Glps) or aquaglyceroporins. The first water channel was isolated from human red blood cell membranes as a novel integral membrane protein of $28 \mathrm{kDa}$ (CHIP28). This channel is now termed aquaporin-1 (AQP1) [3]. Subsequently, thirteen aquaporin paralogs (AQP0-12) have been identified in mammals [1], and up to 38 MIP-related sequences, divided into four types, plasma membrane intrinsic proteins (PIPs), tonoplast intrinsic proteins (TIPs), small and basic intrinsic proteins (SIPs) and nodulin 26-like intrinsic proteins (NIPs), have been documented in plants [4-6]. Each form is composed of a single polypeptide chain varying in length from $\sim 270-350$ amino acids that spans the lipid bilayer six times with three extracellular loops (loop A, C and E) and two intracellular loops (loop B and D), which have their $\mathrm{N}$ - and C-termini located intracellularly. The loops $\mathrm{B}$ and $\mathrm{E}$ fold and extend intramembranous hemi-helices that bear the highly conserved amino acid motifs Asn-Pro-Ala (NPA), which are involved in the formation of the water pore. When translocated to the cell membrane, most aquaporins form homotetramers [7-9], in which one or two monomers may be glycosylated, but each monomer functions as an independent water channel $[10,11]$.

The maintenance of body fluid homeostasis is essential for the survival of any living organism. Aquatic animals also face the problem of direct interaction with their aqueous environment. Depending upon the life history of the species, the osmolarity of the saline or freshwater medium differs manifold from their internal body fluids, and therefore an important physiological role of aquaporins would be expected. Accordingly, some studies in teleosts have reported differential mRNA and protein expression of specific aquaporin isoforms in osmoregulatory organs in response to changes in environmental salinity [12-16]. Moreover, recent studies of neofunctionalized vitellogenins and the essential role of an AQP1-related channel have revealed the evolutionary importance of oocyte hydration in marine teleosts as a pre-adaptation to spawning in the hyperosmotic oceanic environment [17-24]. To date, however, the genomic repertoire of aquaporin isoforms present in teleosts, as well as the permeability properties of the encoded proteins, remains largely unknown.

During the last decades, the zebrafish (Danio rerio) has become a powerful model organism in comparative genomics and developmental biology. This species is amenable for genetic analysis in which large-scale mutagenesis screens have been successfully performed, and a large amount of genomic and expressed sequence tags (ESTs) data, BAC libraries and fine genetic linkage maps have been accumulated [25]. Work with zebrafish has also extended its application to a wide variety of experimental studies relevant to human disease, such as cardiovascular disorders, angiogenesis and neurological and renal diseases [26,27]. Aquaporins have been shown to play important roles in some of these alterations [28], and therefore the diversity and functional properties of zebrafish aquaporins need to be determined prior to using this species as a suitable experimental model in biomedical research and comparative physiology.

With the sequence of the zebrafish genome completed, the complexity of the teleost aquaporin gene family can be assessed. In the present study, we screened the zebrafish genome for aquaporin-related sequences and determined their phylogenetic relationships, permeability properties, and the pattern of mRNA expression in adult tissues. We found a high number of functional aquaporins in this species, and based upon the data obtained we propose a new classification of the piscine aquaporin superfamily.

\section{Results and Discussion}

\section{The zebrafish aquaporin gene family}

The screening of the zebrafish genome revealed the presence of 18 putative members of the aquaporin superfamily, most of them existing as duplicate or triplicate genes, ranging in size from 2.2 to $18 \mathrm{~kb}$ that encode proteins between 255-320 amino acids long (Figure 1). We successfully cloned and characterized 17 of these transcripts. The nomenclature of these genes was chosen based on their phylogenetic position and chromosomal locus, their identity to human orthologs, as well as on their structural and functional features (see below), in accordance with the recommended guidelines for Human Genome Nomenclature [29] and the Zebrafish Information Network [30]. Thus, zebrafish aquaporin-4, $-7,-11 \mathrm{~b}$ and -12 (draqp $4,-7,-11 \mathrm{~b}$ and -12 , respectively) are present as single copy genes, whereas aquaporin-0, $-1,-3,-9$ and -10 genes (draqp0a, $-0 b,-1 a,-1 b,-3 a,-3 b$, $-9 a,-9 b,-10 a$ and $-10 b$, respectively) are duplicates. For the draqp 8 gene, three copies were found (draqp8aa, $-8 a b$ and $-8 b)$. Unlike the other aquaporin isoforms, we found that Aqp8 has both tandem and genomic duplicates encoded within the genomes of zebrafish and stickleback (Gasterosteus aculeatus) (see below for an explanation of the nomenclature).

The deduced amino acid sequences of zebrafish aquaporins contain six predicted transmembrane helices and two NPA boxes that are the hallmark of the MIP 





superfamily [31]. The only exception was DrAqp8b in which only five transmembrane domains could be determined in silico, despite the equality in sequence length. Analysis of the primary structures of the zebrafish aquaporins indicated that they could be classified into two paralogous subgroups and two more divergent subgroups (Figure 2). The first subgroup includes DrAqp0a, $-0 \mathrm{~b},-1 \mathrm{a},-1 \mathrm{~b}$ and -4 , which share $35-85 \%$ amino acid identity (Additional file 1) and clustered with the Escherichia coli aquaporin-Z (EcAqpZ), thus suggesting that these aquaporins belong to the water-specific aquaporin subfamily [31]. The identity of the duplicated isoforms present in this group varied, since DrAqp0a and -0b were $85 \%$ identical, whereas DrAqp1a and -1b share only $61 \%$ identity (Additional file 1 ). The second group includes DrAqp3a, $-3 b,-7,-9 a,-9 b,-10 a$ and $-10 b$, which were $40-73 \%$ identical and clustered together with the E. coli glycerol facilitator (EcGlpF), suggesting that they belong to the aquaglyceroporin (Glp) subfamily. In this group, the identity between DrAqp3a and -3b, DrAqp9a and -9b, and DrAqp10a and -10b, was 73, 61 and $45 \%$, respectively. The percent identity between the aquaporin and Glp groups was as low as $19-28 \%$. The most divergent paralogs were those including the AQP8-like (DrAqp8aa, -8ab and -8b), and DrAqp11b and -12 sequences, which share $23-28 \%$ and $13-25 \%$ identity, and $20-14 \%$ and $4-22 \%$ identity with aquaporins and Glps, respectively. DrAqp8aa, $-8 \mathrm{ab}$ and $-8 \mathrm{~b}$ share $43-60 \%$ identity between them, and DrAqp $11 \mathrm{~b}$ and -12 were $27 \%$ identical. These observations indicate that zebrafish harbours the largest complement of aquaporin genes of any vertebrate studied to date, which can be classified into four tetrapod-like subfamilies [2]: classical water-selective aquaporins $(A Q P 0, A Q P 1$ and $A Q P 4)$, a water and urea transporter $(A Q P 8)$, classical Glps ( $A Q P 3$ and $A Q P 7-10)$, and two unorthodox aquaporins $(A Q P 11 b$ and -12$)$.

\section{Genomic organization}

The division of the zebrafish aquaporin superfamily into four subfamilies inferred from comparison of the deduced protein sequences is mirrored in the intronexon structures (Figure 3). Most classical aquaporins include four exons, draqp4 being an exception with five exons, whereas the Glp genes are characterized by six exons, with the exception of draqp3b, which is encoded by five exons. The draqp $8 a a$, draqp $8 a b$ and draqp $8 b$ transcripts are invariably coded by five exons, whereas the unorthodox draqp12 showed four exons as the aquaporin subfamily. In draqp $11 b$, three exons were observed although in this case the intron-exon boundaries were not well defined and therefore the number of exons could not be determined with certainty. In the majority of zebrafish aquaporin genes the intron lengths were $<5 \mathrm{~kb}$, although the last intron of draqp 4 was of $9.7 \mathrm{~kb}$, whereas the second intron of draqp $9 b$ and draq$p 8 a a$, and the first intron of draqp12, were of 8.2, 6.3 and $6.6 \mathrm{~kb}$ in length. In the case of draqp $9 a$, the quality of the genomic sequences available did not allow assessment of intron length.

Examination of the zebrafish aquaporin gene structures in relation to orthologs in other metazoan organisms revealed a generally well-conserved exon-intron organization (Additional file 2). Classical aquaporins (aqp0, $-1,-2,-5$ and -6$)$ are encoded by 4 exons, with Xenopus tropicalis aqp1 as an apparent exception with 2 exons. Metazoan aqp 4 is encoded by 5 exons, although Drosophila melanogaster aqp4 shows some variability. aqp 8 is a polymorphic gene encoded by 6 exons in tetrapods, 5 exons in teleosts, 2-3 exons in Diptera and 6-7 exons in Nematoda. Classical Glps are highly conserved with 5-6 exons in metazoa, while the vertebrate-specific unorthodox aqp11 has 3 exons, but the more ancestral unorthodox aqp12 retains 3-4 exons in vertebrates, but 4-7 exons in invertebrates.

In silico genomic screening using ensembl v56 [32] also revealed the presence of an 18th gene in zebrafish (ENSDARG00000038202) with an anomalous structure showing 8 exons (Figure 3). The inferred exon structure of this gene is different to that of other metazoan aquaporins and the gene appears to be a hybrid with exons 1-3 more related to AQP2 and -5, but exons 4-8 showing higher nucleotide sequence identity to AQP1 and -6 (Additional file 3). Using BLAST we noted that it was related to tetrapod $A Q P 5$. To validate the existence of this gene, we isolated genomic DNA based upon the predicted ensembl sequence. Using PCR and subsequent sequencing, we found that exons 1-3 are 100\% identical to the predicted sequence, and the gene was therefore





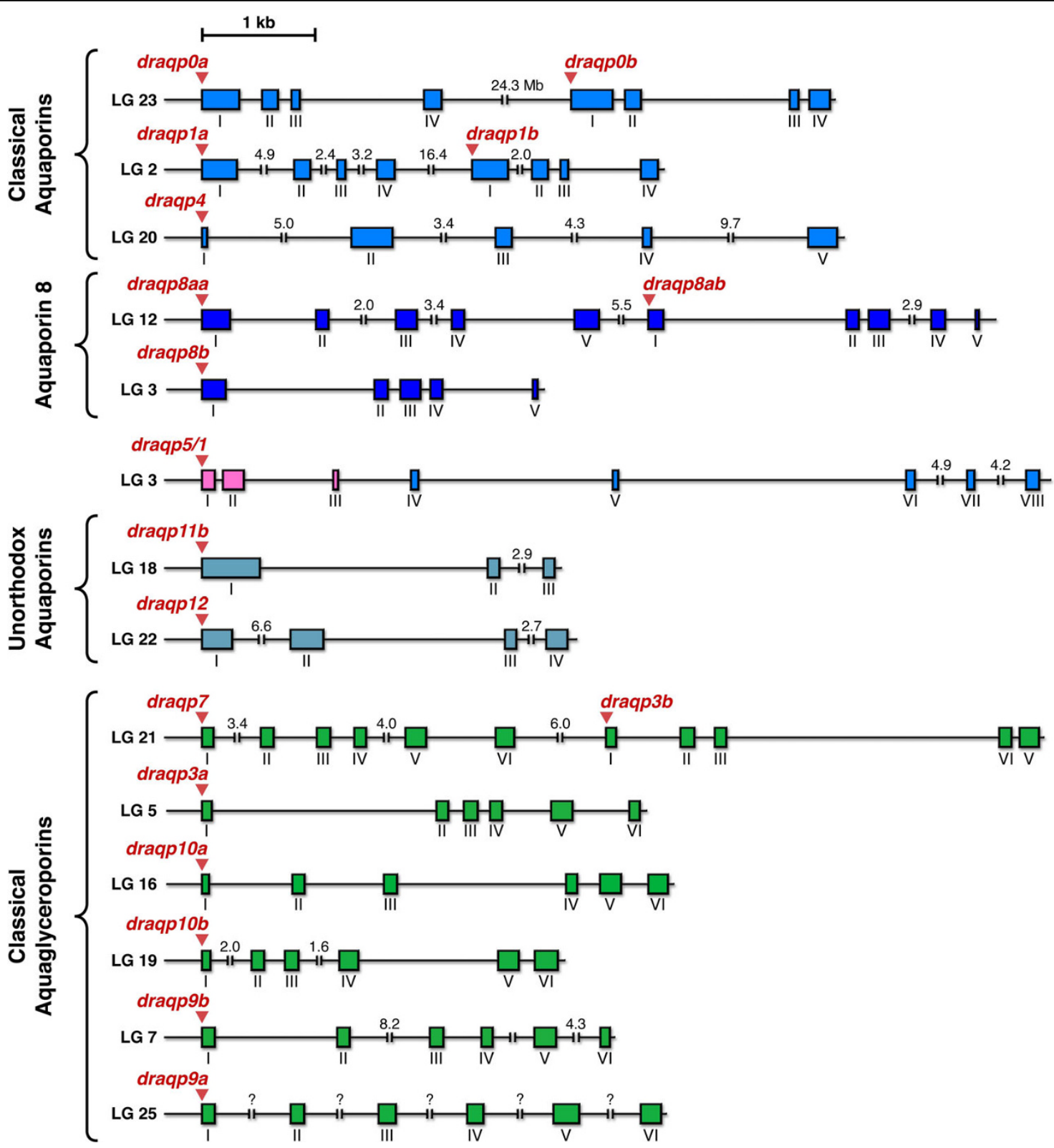

Figure 3 Genomic organization of zebrafish aquaporins. Schematic representation of zebrafish aquaporin gene structures and chromosomal loci. The boxes indicate exons with coding regions only. Distances are in kb or in Mb when indicated. In the case of dragp9a, the quality of the genomic sequence available was insufficient to establish the size of the introns.

named draqp5/1 in accordance with its hybrid status. Maximum likelihood analysis of the codons of draqp $5 / 1$ confirmed that exons 1-3 encode a putative protein that is structurally related to tetrapod AQP5 and -2, while exons 4-8 encode a putative protein that is more related to AQP1 (Additional file 4). Bayesian analysis of the codons placed exon 1-3 basal to $A Q P 0$, but the protein product as a polytomy between AQP5 and AQP2 (data not shown). It therefore appears that despite retaining a large aquaporin repertoire of which $\sim 40 \%$ are duplicate isoforms specific to the teleost crown-clade, the zebrafish lacks functional orthologs of tetrapod $A Q P 2,-5$ or -6 genes.

\section{Phylogenetic analysis of zebrafish aquaporins}

To validate the orthology of the zebrafish aquaporins, we investigated the molecular phylogeny of 233 piscine nucleotide and amino acid sequences in relation to 14 human orthologs (Figures 4 and 5). These analyses corroborated the structural homologies outlined above and 
consistently demonstrated a dual- or in the case of Aqp8 a tri-paralogous clustering of piscine isoforms as sister branches to human orthologs. Two mutually exclusive clades are observed within the superfamily, a vertebrate aquaporin clade containing nine subfamilies (Aqp8, -12, 11, -4, -1, -0, 2, -5 and -6) stemming from EcAqpZ (Figure 4), and a Glp clade containing four vertebrate subfamilies (Aqp9, -3, -7 and -10) that stem from EcGlpF (Figure 5). The elasmobranch aquaporins are represented by single-copy variants, of which all but Aqp7, $-11,-8,-2,-5$ and -6 were found. Each clustered basal to Teleostei or together with the human transcripts indicating that the encoded proteins have differentially evolved functions specific to the actinopterygian or sarcopterygian lineages, respectively. The internal distribution of teleost isoforms within each subfamily was consistent with a whole genome duplication (WGD) event at the root of the crown-clade [20,33-36]. The topology within each teleost subcluster was fully congruent with phylogenetic rank and encompasses members of the Elopomorpha (e.g. eels), Ostariophysi (e.g. zebrafish and carps), Protacanthopterygii (e.g. salmonids) and Acanthomorpha (e.g Gadiformes and Perciformes). We therefore annotated each of the teleost paralogs with the postscript "a" or "b" to reflect the genomic duplicate.

By including genomic variants together with transcripts available from GenBank [37], and by investigating the syntenic relationships of each ortholog (Finn and Cerdà, unpublished data), we were able to validate whether a duplicated gene arose through WGD or resulted from intrachromosomal duplication. Despite the observation that draqp1a and $-1 b$, draqp $0 a$ and $-0 b$, and $d r a q p 7$ and $-3 b$ are linked (Figure 3 ), we only find strong evidence of one tandem duplicate within the crown-clade: aqp8aa (ENSDARG00000045141) and $a q p 8 a b$ (ENSDARG00000071592) in zebrafish, and aqp8aa (ENSGACG00000009127) and aqp8ab (ENSGACG00000014505) in stickleback. One lineagespecific tandem duplicate of aqp10a (aqp10aa: ENSTNIG00000018340 and aqp10ab: ENSTNI G00000018339) was also noted in green-spotted pufferfish (Tetraodon nigroviridis). Both aqp8aa, $-8 a b$ and $a q p 10 a a,-10 a b$ genes are immediately juxtaposed in the genomes of zebrafish (linkage group [LG] 12) and green-spotted pufferfish (LG8), while the WGD products $a q p 8 b$ and $a q p 10 b$ are each located on separate chromosomes, LG3 and LG3, respectively. Unfortunately, it was not possible to characterize the complete aqp 8 loci in other teleosts due to the current location of stickleback $a q p 8 a b$ on scaffold 211, and the absence of $a q p 8 a a,-8 a b$ in green-sptotted pufferfish, and aqp $8 a a$ and $-8 b$ in medaka (Oryzias latipes). For medaka, only the $a q p 8 a b$ gene was found on LG19. However, by comparing our data with the earlier study of Kasahara and colleagues [38], we were able to trace the ancestral chromosome that gave rise to aqp 8 genes in humans and teleosts. Despite multiple rearrangement, fusion and fission events, the major portions of all descendent LGs that harbour vertebrate $a q p 8$ genes can be traced to protochromsome e. For humans this entails LG16, while for Teleostei, the descendent chromosomes are LG2 (predicted locus of $a q p 8 a a,-8 a b)$ and LG3 $(a q p 8 b)$ for green-spotted pufferfish, LG19 (aqp8ab) and LG1 or LG8 (predicted locus of $a q p 8 b$ ) for medaka. The chromosomal loci of aqp8 genes in stickleback and zebrafish further support this ancient origin. Moreover, the early divergence of teleost $a q p 8 a a,-8 a b$ and $-8 b$ is clearly evident in the trichotomous clustering of these transcripts among diverse taxa (Figure 4), and further confirmed through functional and expression analyses in zebrafish (see below).

Similar ancestral reconstructions can be made for the other linked aquaporins, wherein $a q p 1$ genes descend from protochromosome $\mathrm{m}$, aqp 0 originated on prototochromosome 1, and aqp 7 and -3 derive from protochromosomes 1 and $i$, respectively. In this latter instance, $a q p 7$ and -3 have remained linked in all vertebrata, except the opossum (Monodelphis domestica), and thus likely became colocalized prior to the separation of Sarcopterygii from Actinopterygii. The evidence supporting this latter proposal lies in the observation that zebrafish has maintained the linkage between $a q p 3 b$ and -7 on LG21, while $a q p 3 a$ is located on LG5. Zebrafish LG5 is the orthologon of stickleback LGXIII and medaka LG9, both of which harbour the aqp $3 a$ orthologs. Hence the single $a q p 7$ gene found in teleost genomes is the surviving $a q p 7 b$ product of WGD. The close linkage of teleost $a q p 1 a$ and $-1 b$ genes, suggests that they could have arisen through tandem duplication. However, the fact that all teleost genomes, except medaka, retain both isoforms, and that each shows dichotomous clustering in the phylogenetic trees (Figure 4), their origin must be close to, or coincident with the WGD event at the root of the crown-clade. Hence, although we cannot exclude a tandem duplication scenario suggested earlier [22], a parsimonious explanation for the appearance or aqp $1 a$ and $-1 b$ would seem to be WGD with subsequent colocalization, rather than local duplication and loss of WGD paralogs. The divergence of these isoforms is clearly evident in the phylogenetic trees, where $a q p 1 b$ transcripts display significantly longer branch lengths compared to the aqp1a paralogs. This latter feature is consistent with our earlier findings wherein Aqp1b is a rapidly evolving channel protein with novel functions specifically associated with oocyte hydration [17-22] and osmoregulatory processes [16].

In a separate analysis of metazoan aquaporins (Finn and Cerdà, unpublished data) we confirmed that $a q p 7$, 




Figure 4 Bayesian majority rule consensus tree for the codon alignment of piscine and human aquaporins. The upper right panel shows the summarized topology of the complete tree rooted with archaean aqpm. The left panel shows the topology of the classical aquaporins (aqp0, -1 , and -4), unorthodox aquaporins (aqp11 and -12) and Aqp8. Accession numbers are annotated with the taxa. Bayesian posterior probabilities are shown at each node. Scale bar indicates the rate of expected nucleotide substitutions per site. 


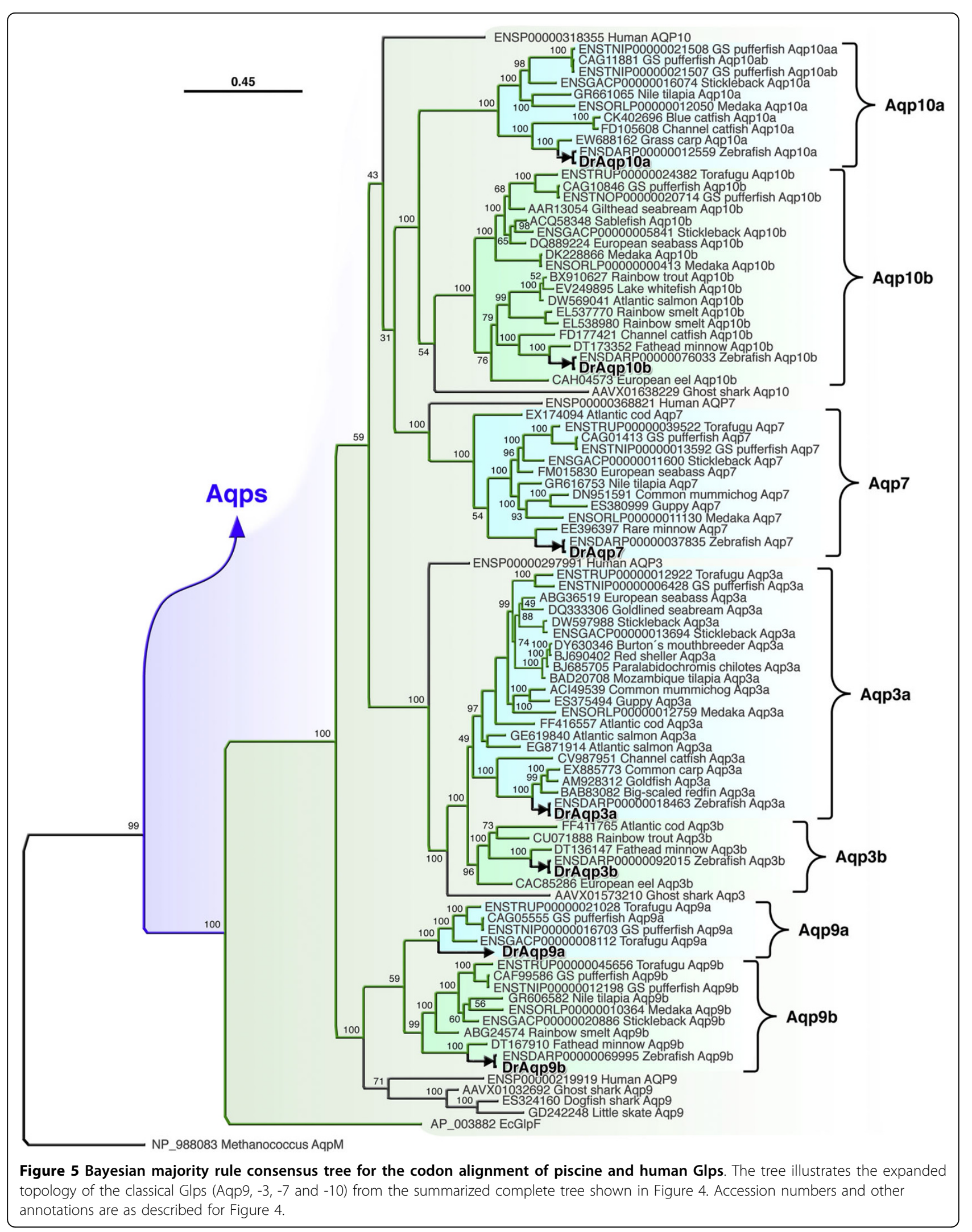


-12 and -4 have remained single-copy genes in nonhuman vertebrates while the present data show that Aqp11 duplicated at the root of the teleost crown-clade. With the exception of torafugu (Takifugu rubripes), which retains both aqp11a and $-11 b$, Teleostei, including zebrafish, appear to have differentially retained alternate isoforms. This is clearly shown in the phylogenetic tree (Figure 4), with closely related Protacanthopterygii and Acanthomorpha harbouring opposite isoforms. Based upon the more ancestral ostariophysan position of zebrafish and the fathead minnow (Pimephales promelas), we arbitrarily assigned the zebrafish cluster as the "b" variant.

Although zebrafish retains two isoforms of $a q p 3$, these genes proved the most difficult class of Glp to resolve (Figure 5). Here we show the codon topology, which also includes variants obtained from EST databases. By specifically increasing the taxon sampling of these Glps, we were able to resolve a branch topology that matched the chromosomal loci of the teleost genes. As a result, the genomic duplicates are annotated as "a" or "b" accordingly. However, the protein trees did not always corroborate the codon topology and therefore these Glps will require further validation with the advent of new sequence data. The placement of draqp5/1 as an outgroup between $a q p 0$ and -1 was consistent with its hybrid status, while the $A Q P 2,-5$ and -6 genes are specific to the sarcopterygian lineage.

\section{Molecular features of zebrafish aquaporins and functional implications}

The characteristic residues in a water channel that distinguish a true water facilitator from a Glp have previously been analyzed by comparing sequences of aquaporins with known functions [39]. That comparison resulted in the identification of five invariant or nearly invariant residues (P1 to P5) in aquaporins and Glps on the basis of 153 sequences ranging from bacteria to humans (Figure 6A,B,D,E). However, structural and functional studies of AQP1 and GlpF suggest the presence of two constriction sites in the water pore, in addition to the P1-P5 residues, that underlie their high selectivity and efficiency with regard to water or glycerol transport [9,40-43] (Figure 1).

The first constriction is formed by the opposing NPA motifs located at each positive end of $\alpha$-helices 3 and 7 (on loops B and E; Figure 6A,B,D,E), such that the Asn creates an electrostatic barrier in this region [44]. Together with desolvation, these regions are essential for water transport specificity while excluding proton transport [44]. Accordingly, Asn is the least variable in aquaporin sequences whereas Pro and Ala are more exchangeable $[2,45]$. The primary structure of zebrafish aquaporins and Glps confirm these observations since
Asn was conserved in both NPA boxes of all paralogs, whereas Pro is substituted by Ala in the first box of DrAqp7 as observed in human and rat AQP7 (Figure 6F). The third residue of the NPA boxes shows more variation as previously found in over 450 aquaporinencoding genes [2]. In zebrafish, Ala is substituted by Pro (first box of DrAqp8ab), Ser (first box in DrAqp8b), Val (second box in DrAqp8b), or Thr (first and second box in DrAqp3b, and DrAqp7 and -11b, respectively).

The second significant energy-barrier in aquaporins is located close to the extracellular exit of the channel forming the narrowest region of the pore and is referred to as the aromatic/arginine $(\mathrm{ar} / \mathrm{R})$ constriction $[46,47]$. This region in water-selective aquaporins is formed by four amino acids (Phe ${ }^{56}$, His ${ }^{180}, \mathrm{Cys}^{189}$ and $\mathrm{Arg}^{195}$ in human AQP1; Figure 6B,C,E,F) that create the hydrophobic and size filter [48]. The site sensitive to mercurial inhibition (Cys ${ }^{189}$ in AQP1) [11] is less conserved. In contrast, in GlpF, and essentially in all other Glps, the ar/ $\mathrm{R}$ region is wider and more hydrophobic due to the lack of His and substitution of the Cys by a second aromatic residue, which allows the passage of polyols and urea, and possibly of other small solutes such as $\mathrm{NH}_{3}$, $\mathrm{CO}_{2}$ or $\mathrm{O}_{2}$ [48].

The present data show that zebrafish aquaporins previously inferred to be water-selective or Glps after amino acid sequence analysis retain the respective three amino acid consensus in the ar/R constriction, and show the P1-P5 residues conserved in each sub-family (Figure $6 \mathrm{C}, \mathrm{F}$ ). However, the Cys residue just prior to the second NPA box, which is potentially mercury-sensitive, is maintained in DrAqp1a and -1b. Similarly, DrAqp8aa, $-8 \mathrm{ab},-8 \mathrm{~b},-9 \mathrm{a},-9 \mathrm{~b}$ and $-10 \mathrm{a}$ also show a Cys upstream of the second NPA motif in loop B but at slightly different positions. Despite these minor differences, the structural features of zebrafish aquaporins strongly suggest that they encode functional channels. A slightly different situation is found in DrAqp8aa, -8ab and $-8 \mathrm{~b}$, which despite having the conserved P1-P5 residues of the aquaporin subfamily, contain His instead of $\mathrm{Phe}^{56}$, and Val or Ile instead of His ${ }^{180}$, in the ar/R constriction (Figure 6C). As for their mammalian counterparts, DrAqp11b and -12 appear to be more divergent since they did not show any of the typical residues in the ar/ $\mathrm{R}$ constriction and only three aquaporin-conserved amino acids (P2, P4 and P5) (data not shown).

\section{Water and solute permeability of zebrafish aquaporins}

Typically, aquaporin water-channel activity is tested by the oocyte-swelling assay [3], in which Xenopus laevis oocytes expressing aquaporins are exposed to hypoosmotic shock and the subsequent water influx is measured by determining volume changes over time. Solute permeability can also be determined volumetrically in 
A

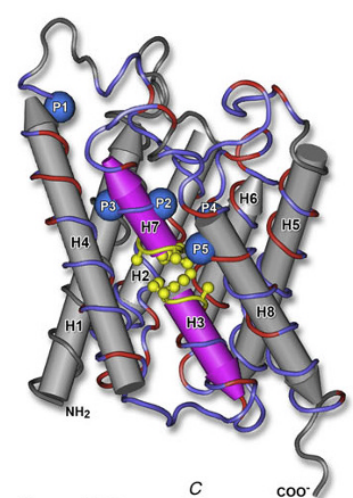

B

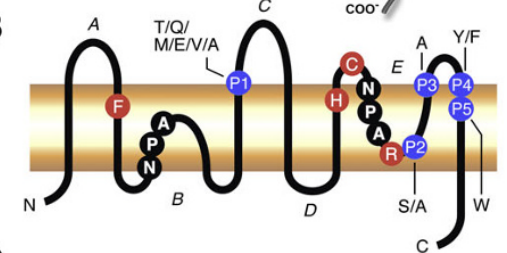

C

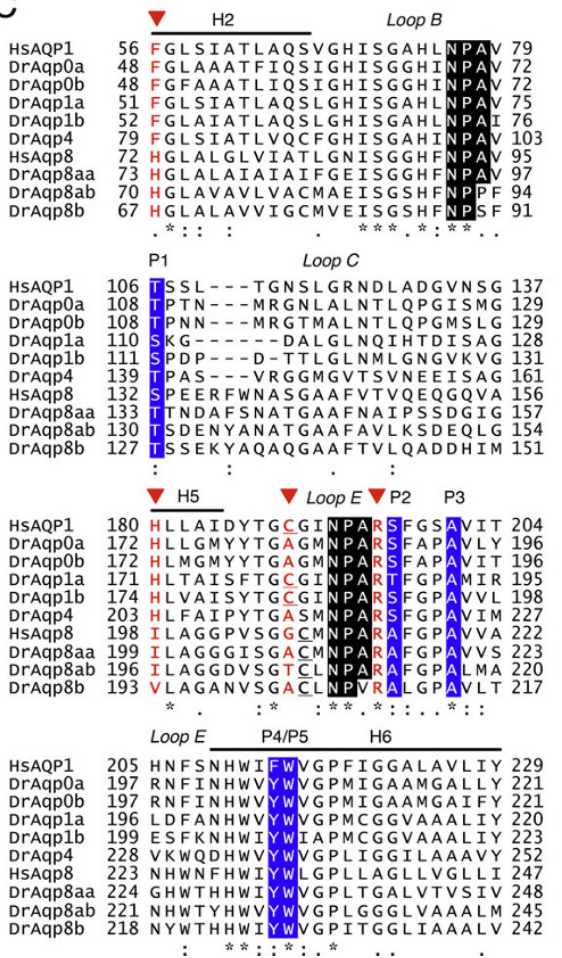

D

E
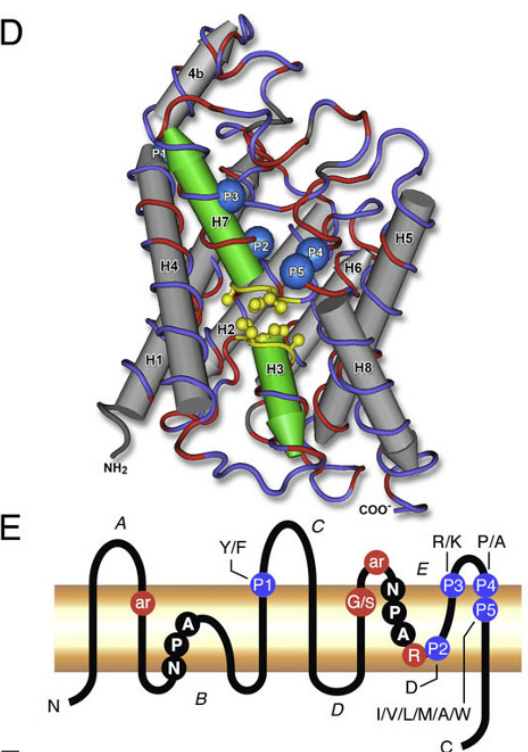

$\mathrm{F}$

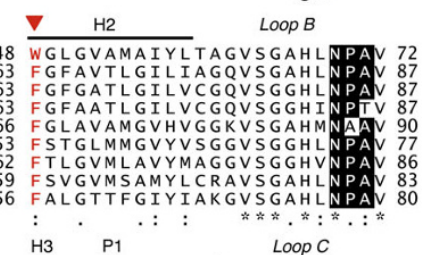

H3 P1

Loop C

ECG7pF 103 LVYGLYYNLFFDFEQTH--HIVRG 124

DrAqp3a 118 IIFAEYHDAIYDYAGESNELLVLG 142

DrAqp3b 118 IIFGMYFDAIWKFGQGS--LDVDG 140

DrAqp7 121 TIFSLYYDAINHFCGGN-- LTVSG 143

DrAqp9a 108 AVFGLYYDAFMEF

DrAqp10a 114 LVYLIYHDAIMEFSGGV--LTVFG 136

DrAqp10b 111 TVALQYYDAIMDFTGGH--LTVSG 133

$$
\nabla \text { H5 }
$$$$
\therefore \text {. }
$$

P3

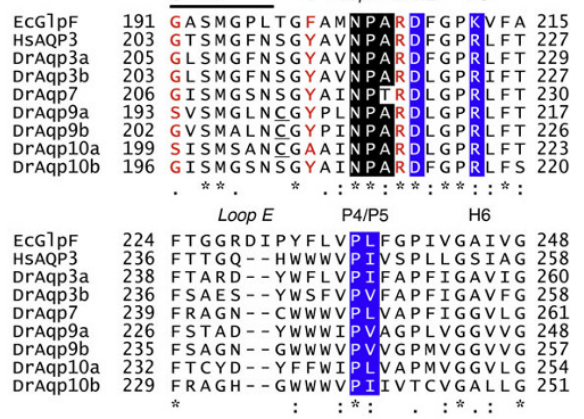

Figure 6 Structural features of zebrafish aquaporins. (A-C) Water-selective aquaporins and aquaglyceroporins (Glps; D-F). (A and D) Threedimensional reconstruction of DrAqp4 wrapped to the crystallographically resolved structure of Escherichia coli AqpZ (1RC2 chain B), and DrAqp3a wrapped to the crystallographically resolved structure of E. coli GlpF (1LDI chain A). Molecules are mirror tube-worm renders rotated to show identical (red) and non-identical (blue) residues and the annotated features including the blue space-filled conserved sites (P1-P5) and the opposing yellow ball and stick Asn-Pro-Ala (NPA) motifs between hemi-helices H3 and H7. Despite low primary identity/similarity (21.1/36.6\% for DrAqp4; 34.1/53.0\% for DrAqp3a) the secondary and tertiary structures appear conserved. (B and E) Schematic diagram of aquaporin monomers showing the 6 transmembrane helices $(\mathrm{H})$, the two NPA motifs, the amino acids forming the aromatic/arginine (ar/R) constriction, and the five residues (P1-P5) conserved in water-selective (B) and Glps (E). In each position the conserved residues are indicated. (C) Amino acid sequence alignment of human AQP1 and AQP8 (HsAQP1 and HsAQP8), mouse AQP8 (MmAQP8), DrAqp0a, -0b, -1a, -1b, -4, and zebrafish AQP8-related sequences (DrAqp8aa, -8ab and -8b). The arrowheads point to the positions of the ar/R constriction. The P1-P5 conserved amino acids are shaded in blue. The asterisks indicate identical residues, whereas conserved amino acid substitutions and substitutions with similar amino acids are indicated by a double or single dot, respectively. The potential mercury-sensitive Cys site before the second NPA motif is underlined. (F) Amino acid sequence alignment of EcGlpF, human AQP3 (HsAQP3) and zebrafish Glps. Symbols and notes as in C. 
isotonic solutions, or by employing radiolabeled compounds $[49,50]$. Therefore, to determine experimentally the substrate specificity of the zebrafish aquaporins, the cloned cDNAs were expressed in $X$. laevis oocytes.

Expression of all of the aquaporin isoforms, except draqp $11 b$ and -12 , induced a three- to twenty-fold increase in oocyte water permeability (Figure 7). Interestingly, however, water permeability of oocytes expressing draqp $3 a$ and $-3 b$ was reduced by acidic $\mathrm{pH}$, as found for mammalian AQP3 [51] and European eel (Anguilla anguilla) Aqp3 [52], with maximum permeability occurring at $\mathrm{pH}$ 8.5. Swelling (data not shown) and isotope-labeled solute uptake assays demonstrated that oocytes injected with $\operatorname{draqp} 3 a,-3 b,-7,-9 a,-9 b$, $-10 a$ and $-10 b$ were also permeable to glycerol and urea, although in the case of draqp10b urea permeability was low (Figure 7). Oocytes expressing draqp8aa or $-8 a b$ were permeable to water and urea, but not glycerol. In contrast, oocytes expressing draqpOa, $-0 b,-1 a,-1 b$ and -4 , as well as draqp $8 b$, were not permeable to either of these solutes.

Water and glycerol transport through most aquaporins was significantly $(p<0.01)$ blocked by $0.3 \mathrm{mM} \mathrm{HgCl}_{2}$ even in the absence of Cys ${ }^{189}$ in their deduced amino acid sequences (Figure 6C,F). Such inhibition was not always reversed with the reducing agent $\beta$-mercaptoethanol. However, DrAqp4 (as in mammalian AQP4) and DrAqp8b were mercury-insensitive, even though DrAqp8b has a potential mercury-sensitive Cys upstream of the second NPA box (Figure 6C). It thus appears that water and solute flux through zebrafish aquaporins can be blocked by mercurial compounds regardless of the presence or absence of Cys ${ }^{189}$. These observations have also been noted for rat AQP3 [53,54] and plant aquaporins $[55,56]$. The present findings suggest that sensitivity of zebrafish aquaporins to mercurials is a complex phenomenon, as has been suggested for other aquaporins, and may involve other residues in addition to $\mathrm{Cys}^{189}$ [56].

Expression of draqp11b or -12 had no effect on oocyte water (Figure 7), or glycerol (data not shown) permeability. The absence of water and solute transport in $X$. laevis oocytes expressing mammalian AQP11 and -12 has been previously reported $[57,58]$. For AQP12, this seems to be caused by the absence of protein expression in the oocyte plasma membrane [57], but in the case of AQP11 the protein is readily targeted to the plasma membrane [58]. The underlying mechanisms involved in the functional failure of AQP11 and -12 in oocytes are not well known, but may be related to the fact that these aquaporins are localized intracellularly in vivo $[57,59]$. However, when AQP11 was reconstituted into liposomes this protein proved to be a functional water channel [60]. Although the identity of DrAqp11b and

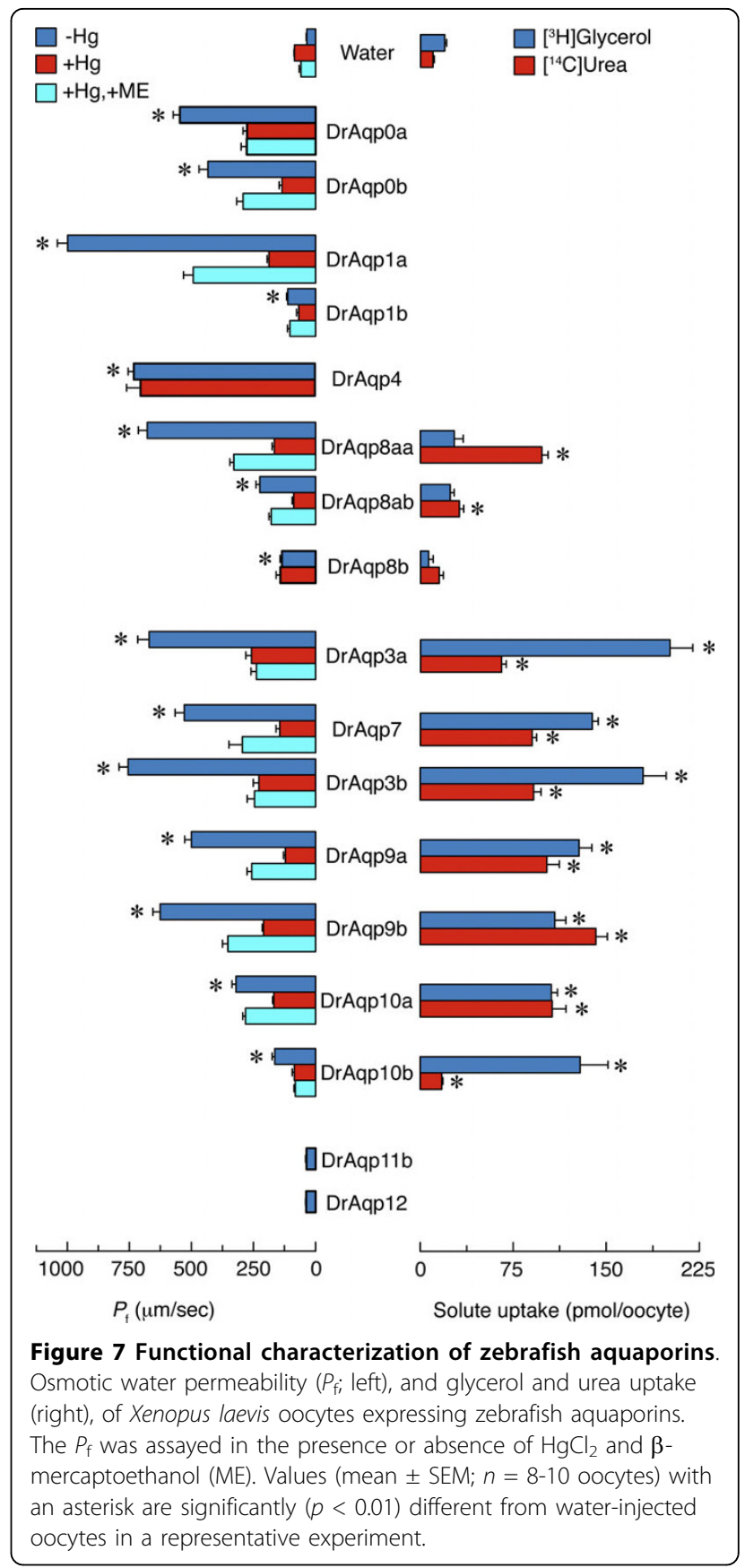

-12 with AQP11 and -12 is low (35\% and 38\% identity, respectively), a similar situation may be speculated in zebrafish. Further studies employing reconstitution of DrAqp11b and -12 into proteoliposomes, as well as specific antibodies to elucidate their subcellular localization, would help to clarify this issue.

\section{Expression pattern of zebrafish aquaporins}

The relative expression of the zebrafish aquaporin genes in adult tissues was evaluated by RT-PCR employing 
isoform-specific oligonucleotide primers (Figure 8). These data revealed that while all genes except draqp5/ 1 were expressed, some aquaporin transcripts were ubiquitiously distributed in the tissues examined or appeared to be tissue-specific. Notably, mRNAs derived from duplicated paralogs showed slightly different expression patterns, although redundancy in some tissues was also observed.
Only two zebrafish aquaporin transcripts (draqp1a and -12) showed tissue-wide expression patterns. The ubiquitous expression of draqpla transcripts is consistent with the presence of the mammalian AQP1 ortho$\log$ in endothelial barriers of almost all tissues and in many epithelia [61]. Its presence in the zebrafish gastrointestinal tract and gills agrees with that reported in other teleosts $[12,15,16,62]$. In contrast, expression of
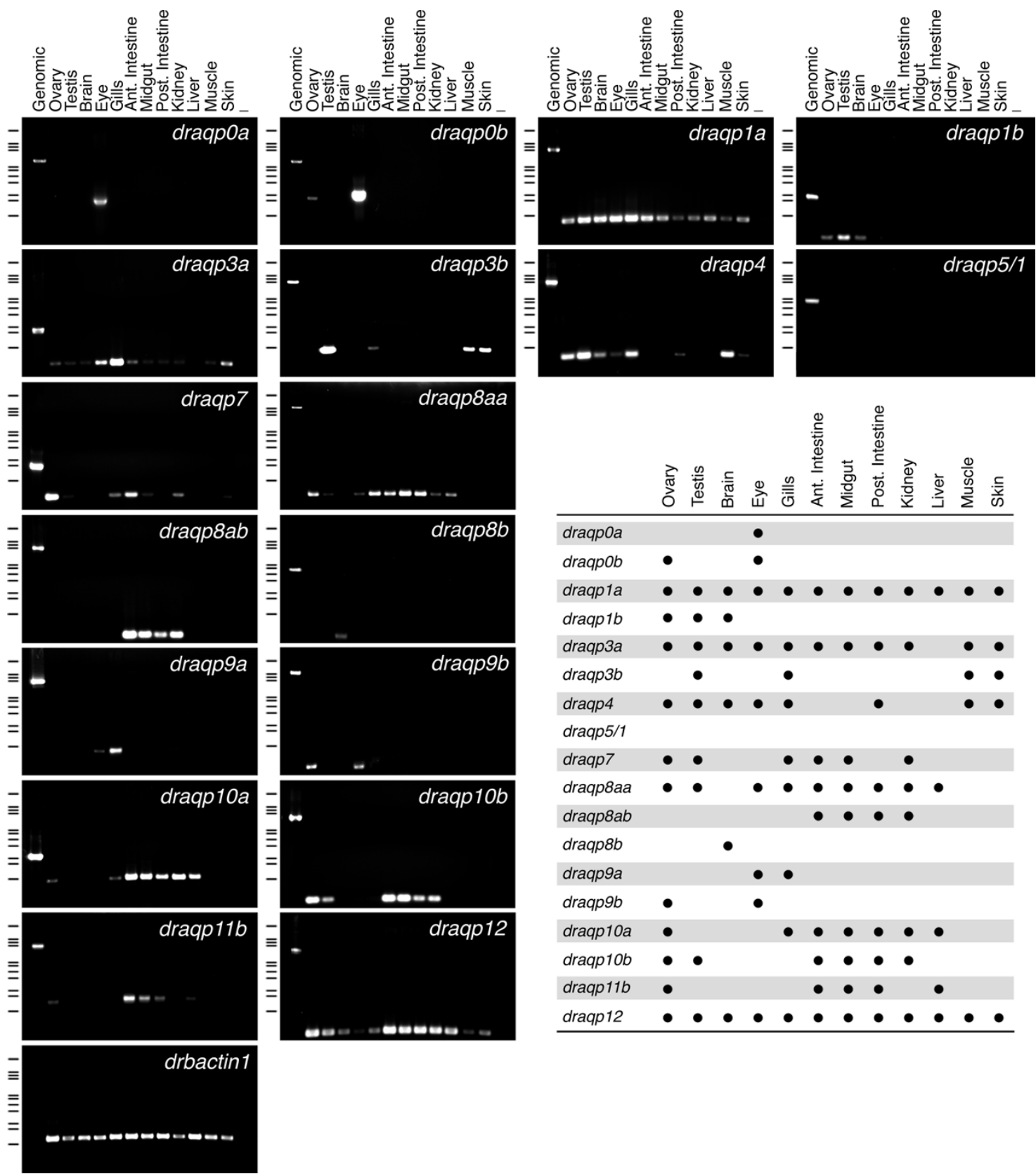

Figure 8 Aquaporin gene expression in adult tissues of zebrafish. Representative RT-PCR analysis of aquaporin and b-actin 1 (drbactin 1) transcripts. PCR on genomic DNA was used as control. Minus indicates absence of RT during CDNA synthesis. The size (kb) of PCR products and molecular markers are indicated on the left; from top to bottom: $21.23,5.15,4.27,3.53,2.03,1.91,1.58,1.37,0.95,0.83$ and 0.56 . A summary of the presence or absence of the aquaporin transcripts in the different tissues is shown to the lower right. 
draqp $1 b$ was restricted to the ovary, testis and brain, as previously reported [22]. The ubiquitous expression of the unorthodox aquaporin draqp12, with lower levels in the eye and muscle, differs markedly from the exclusive expression of mammalian AQP12 in the pancreatic acinar cells [57] and the retina [63]. The vertebrate-specific unorthodox aquaporin, draqp11b, also showed a different expression pattern compared to that described for mammalian AQP11. In mice, kidney expression of AQP11 seems to be essential during development since either its absence or mutations of the amino acid sequence induce renal failure $[59,64]$. In our PCR experiments, however, we could not observe detectable levels of draqp $11 b$ transcripts in the kidney, although they were abundantly expressed in the gut and were also detected in ovary and liver.

In contrast to draqp1a and -12, transcripts of draqp0a and $-8 b$ were exclusively detected in tissues of the eye and brain, respectively, and $d r a q p 0 b$ was also observed in the ovary. The specific expression of draqp 0 paralogs in the eye is consistent with that previously described in the common mummichog (Fundulus heteroclitus) $[65,66]$, as well as in mammals where AQP0 is predominantly present in fibre cells of the lens [67]. Expression of draqp $8 b$ was noted only in the brain, where draqp $8 a a$ and $-8 a b$ were not expressed. In contrast, these latter transcripts were abundantly expressed in the gut and kidney, draqp8ab mRNA being also present in the ovary, testis, eye, gills and liver. The diffuse expression of draqp $8 a a$ and $-8 a b$ resembles that found for mammalian AQP8, which is present in the testis, ovary, kidney, liver, pancreas, small intestine and colon $[1,68]$. The expression of draqp4 is similar to that of mammalian AQP4 [1], which is distributed in the brain, small intestine and muscle, but also in kidney. In the present study, however, we did not detect draqp 4 transcripts in the zebrafish kidney.

The distribution of classical Glp mRNAs in zebrafish was comparable to that described for mammals and some teleosts [69,70]. draqp3a showed the most extensive distribution in all tissues examined except in the liver, showing high abundance in gills as previously found for other teleosts [14,15]. In contrast, draqp $3 b$ transcripts were seen only in the testis, gills, muscle and skin. The draqp $9 a$ and $-9 b$ isoforms, as well as draqp10a and $-10 b$, also showed differential expression in some tissues. The expression of two functional isoforms of draqp10 in zebrafish, however, differs from that reported for mouse in which AQP10 was suggested to be a pseudogene [71]. As in mammals, draqp 7 is expressed in the gonads and the kidney, while the expression observed in the intestine may reflect an adipocyte function [72,73].
The RT-PCR studies suggest that redundant expression of duplicated aquaporin isoforms occurs in many tissues of zebrafish. The physiological significance of these findings is intriguing. In other teleosts, such as the gilthead seabream (Sparus aurata), redundant expression of aqp1a (saaqp1a) and $-1 b$ (saaqp1b) in the ovary and gastrointestinal tract is also observed, but immunological studies have revealed that their protein products are differentially localized [16,17]. Thus, in the ovary SaAqp1b is oocyte-specific, whereas SaAqp1a seems to be only present in the external epithelium surrounding the ovarian follicle. In the gut, SaAqp1b is exclusively detected at the apical membrane of rectal enterocytes, whereas SaAqp1a is localized at the brush border of enterocytes in the duodenum and hindgut. Therefore, the significance of the redundant aquaporin expression in zebrafish must be further investigated by determining the subcellular sites of transcription and protein targeting.

\section{Conclusions}

In this study, we identified a large number of MIP encoding genes in zebrafish when compared to tetrapods. By integrating the molecular phylogeny and the genomic loci with structural and functional analyses we demonstrate dual- or tri-paralogy between teleost aquaporins and human orthologs. Consequently teleost aquaporins can be classified into the same water-selective and Glp subfamilies previously described in vertebrates [2]. Expression in X. laevis oocytes also demonstrated that zebrafish aquaporin genes, except $a q p 11 b$ and -12 , encode functional channels, which in most cases have retained the substrate specificity of the tetrapod counterparts. Some mammalian aquaporins are also permeable to $\mathrm{NH}_{3}, \mathrm{CO}_{2}$ or $\mathrm{O}_{2}$ [74], but whether any of the zebrafish orthologs are permeable to these compounds remains to be investigated.

The high number of aquaporin genes in zebrafish and other teleosts result from WGD at the root of the crown-clade. Most duplicated isoforms are retained, while aqp $4,-7$, and -12 have remained single-copy genes in non-human vertebrates. The findings further reveal that tandem duplication has occurred within the clade and in a lineage-specific manner, but such intrachromosomal replication events are rare for this superfamily. While most of the duplicated genes seem to be conserved during the diversification of teleosts, some such as aqp 11, -3 and -8 appear to have been differentially retained among species. The reasons for the evolutionary selection of specific aquaporin isoforms in teleosts, as well as the potential neofunctionalization of others, as shown for $a q p 1 b$ in the hydrating oocytes of marine teleosts $[17,22]$, have yet to be determined. 
An interesting finding was that zebrafish harbours a hybrid gene with partial structural identity to the tetrapod $A Q P 2, A Q P 5$ and $A Q P 6$ orthologs. This hybrid sequence is not expressed, and it appears to represent a pseudogene. The physiological significance of this observation remains to be elucidated. The present study, however, provides a validated nomenclature for the piscine aquaporin superfamily and lays the foundation for further functional studies in zebrafish.

\section{Methods}

\section{Fish}

Adult zebrafish were purchased from local pet stores and maintained as described [75]. To obtain samples of the different tissues, fish were sedated by immersion for approximately $15 \mathrm{~min}$ in $80-100 \mathrm{ppm}$ phenoxyethanol, and sacrificed by decapitation. Tissue samples were immediately processed for RNA extraction or flash-frozen in liquid nitrogen and stored at $-80^{\circ} \mathrm{C}$. Procedures relating to the care and use of animals were approved by the Ethics Committee from IRTA in accordance with the Guiding Principles for the Care and Use of Laboratory Animals.

\section{Genome and transcript analysis}

Zebrafish aquaporin coding sequences were identified by basic local alignment search tool (BLAST) [76] homology searches of the NCBI [37] and ensembl [32] databases with mammalian and previously cloned teleost aquaporin sequences. The predicted amino acid sequences were extracted, analyzed using BLASTP, and annotated according to the nomenclature established for zebrafish [30]. Intron-exon splicing sites were verified visually using available cDNA sequences and/or corresponding ESTs, and genomic sequences. Potential transmembrane helices in the encoded amino acid sequences were predicted using the SOSUI engine v1.11 [77], TMHMM Server v2.0 [78] and Phobius [79], or via molecular wraps to resolved aquaporin structures (Additional file 5).

\section{cDNA Cloning}

In this study, cDNAs bearing the complete coding region of draqp0a, $-0 b,-3 a,-3 b,-4,-7,-8 a a,-8 a b,-8 b$, $-9 a,-9 b,-10 a,-10 b,-11 b$, and -12 were cloned by conventional RT-PCR. Full-length draqp $1 a$ and $-1 b$ cDNAs were previously isolated $[17,22]$. Total RNA was extracted from the kidney, gut, gills, liver, brain and ovary of adult fish using the RNeasy Maxikit (Qiagen) followed by DNase treatment following the manufacturer's instructions. A pool of 1-10 $\mu \mathrm{g}$ total RNA from the different tissues was reverse transcribed using $0.5 \mu \mathrm{g}$ oligo $(\mathrm{dT})_{17}, 1 \mathrm{mM}$ dNTPs, 40 IU RNAse inhibitor (Roche), and $10 \mathrm{IU}$ MMLuV-RT enzyme (Roche), for $1.5 \mathrm{~h}$ at $42^{\circ} \mathrm{C}$. For amplification, PCR was carried out with $1 \mu \mathrm{l}$ of the synthesized cDNA and specific oligonucleotide primers designed according to the available cDNAs and genomic sequences. The PCR reactions were conducted using a total volume of $50 \mu \mathrm{l}$ containing $1 \mathrm{x}$ reaction buffer supplemented with 1.5 $\mathrm{mM} \mathrm{MgCl} 2,0.2 \mathrm{mM}$ dNTPs, $0.2 \mathrm{mM}$ of each forward and reverse primer, and $1 \mathrm{U}$ of Easy-ATM High-Fidelity PCR Cloning Enzyme (Stratagene). The PCR conditions were an initial denaturation at $95^{\circ} \mathrm{C}$ for $5 \mathrm{~min}$, followed by 36 cycles at $95^{\circ} \mathrm{C}$ for $1 \mathrm{~min}, 60-65^{\circ} \mathrm{C}$ for 1 min (depending on the $\mathrm{Tm}$ of each primer pair), and $72^{\circ} \mathrm{C}$ for $1 \mathrm{~min}$. A final elongation cycle at $72^{\circ} \mathrm{C}$ for 7 min was carried out in all cases. The products were cloned into the pGEM-T Easy Vector (Promega) and both strands sequenced by BigDye Terminator v3.1 cycle sequencing on ABI PRISM 377 DNA analyzer (Applied Biosystems). The deduced amino acid sequences of cloned cDNAs were $100 \%$ identical to those available in the databases, except for DrAqp7 and $-11 \mathrm{~b}$, which were $98 \%$ identical (3 and 2 amino acid changes, respectively), and the cDNAs encoding DrAqp8aa, $-8 \mathrm{ab},-9 \mathrm{~b},-10 \mathrm{a}$ and -12 , which were $99 \%$ identical ( 1 amino acid change in each sequence). In further structural and phylogenetic analyses we used the amino acid sequences derived from the cDNAs cloned in this study since they were found to encode functional proteins in oocytes.

\section{Functional expression in Xenopus laevis oocytes}

Zebrafish aquaporin full-length cDNAs were cloned into the EcoRV/SpeI sites of the oocyte expression vector pT7Ts [80]. Capped RNAs (cRNAs) were synthesized in vitro with T7 RNA Polymerase (Roche) from XbaI-linearized pT7Ts vector containing the different aquaporin cDNAs. The isolation and microinjection of stage $\mathrm{V}-\mathrm{VI}$ oocytes was performed as described previously [80]. Oocytes were injected with $50 \mathrm{nl}$ of distilled water (negative control) or $50 \mathrm{nl}$ of water solution containing 1-10 ng cRNA.

\section{Swelling assays}

The osmotic water permeability $\left(P_{\mathrm{f}}\right)$ was measured from the time course of oocyte swelling in a standard assay. Oocytes were transferred from 200 mOsm modified Barth's culture medium (MBS; $0.33 \mathrm{mM} \mathrm{Ca}\left(\mathrm{NO}_{3}\right)_{2}, 0.4$ $\mathrm{mM} \mathrm{CaCl} 2,88 \mathrm{mM} \mathrm{NaCl}, 1 \mathrm{mM} \mathrm{KCl}, 2.4 \mathrm{mM}$ $\mathrm{NaHCO}_{3}, 10 \mathrm{mM}$ Hepes, $0.82 \mathrm{mM} \mathrm{MgSO}$, $\mathrm{pH}$ 7.5) to $20 \mathrm{mOsm} \mathrm{MBS}$ at room temperature. Oocyte swelling was followed by video microscopy using serial images at $2 \mathrm{~s}$ intervals during the first $20 \mathrm{~s}$ period. For Draqp3a and $-3 \mathrm{~b}$, the swelling assays were performed at $\mathrm{pH} 8.5$. The $P_{\mathrm{f}}$ values were calculated taking into account the time-course changes in relative oocyte volume $\left[\mathrm{d}\left(\mathrm{V} / \mathrm{V}_{\mathrm{o}}\right) /\right.$ 
$\mathrm{dt}]$, the molar volume of water $\left(\mathrm{V}_{\mathrm{w}}=18 \mathrm{~cm}^{3} / \mathrm{ml}\right)$ and the oocyte surface area $(\mathrm{S})$ using the formula $\mathrm{V}_{\mathrm{o}}[\mathrm{d}(\mathrm{V} /$ $\left.\left.\mathrm{V}_{\mathrm{o}}\right) / \mathrm{dt}\right] /\left[\mathrm{SV}_{\mathrm{w}}\left(\mathrm{Osm}_{\mathrm{in}}-\mathrm{Osm}_{\text {out }}\right)\right]$. To examine the inhibitory effect of mercury on $P_{\mathrm{f}}$, oocytes were pre-incubated for $15 \mathrm{~min}$ in MBS containing 0.5 or $0.3 \mathrm{mM} \mathrm{HgCl}_{2}$ before and during the swelling assays. To determine the reversibility of the inhibition, the oocytes were rinsed 3 times with fresh MBS and incubated for another $15 \mathrm{~min}$ with $5 \mathrm{mM} \beta$-mercaptoethanol before being subjected to swelling assays.

\section{Radioactive solute uptake assays}

To determine the uptake of $\left[{ }^{3} \mathrm{H}\right]$ glycerol $(60 \mathrm{Ci} / \mathrm{mmol})$ and $\left[{ }^{14} \mathrm{C}\right]$ urea $(52 \mathrm{mCi} / \mathrm{mmol})$ groups of 10 oocytes, injected with water or cRNA, were incubated in $200 \mu \mathrm{l}$ of MBS containing $20 \mu \mathrm{Ci}$ of the radiolabeled solute (cold solute was added to give $1 \mathrm{mM}$ final concentration) at room temperature. After $10 \mathrm{~min}$ (including zero time for subtraction of the signal from externally bound solute), oocytes were washed rapidly in ice-cold MBS three times, and individual oocytes were dissolved in 5\% SDS for scintillation counting.

\section{Statistical analysis of $P_{\mathrm{f}}$ and solute uptake}

Data are expressed as mean \pm SEM. The data shown are from a representative experiment out of 3-4 different trials producing similar results. The measured values of $P_{\mathrm{f}}$, and glycerol and urea uptake were statistically analyzed in an unpaired Student's $t$ test; $p$ values $<0.01$ were considered significantly different.

\section{Gene expression analysis}

Total RNA was extracted from different tissues, ovary, testis, brain, eye, gills, anterior intestine, midgut, posterior intestine, kidney, liver, muscle and skin, of 5-10 adult fish using the RNeasy Mini Kit (Qiagen). An aliquot of RNA (500 ng) was treated with Turbo-DNase (Ambion) and reverse transcribed as described above. PCR was carried out with 0.3-0.6 $\mu$ l of cDNA employing Tfi DNA Polymerase (Invitrogen) and aquaporin isoform-specific oligonucleotide primers (Additional file 6). For each aquaporin nucleotide sequence, the oligos were designed to flank one or separated introns that were complementary to non-conserved regions among aquaporin paralogs. The reactions were carried out in a $25 \mu \mathrm{l}$ volume containing $1 \mathrm{x}$ reaction buffer, $1.5 \mathrm{mM} \mathrm{MgCl}, 0.2 \mathrm{mM}$ dNTPs, $0.2 \mathrm{mM}$ of each primer and $1 \mathrm{U}$ of $T f i$ polymerase. The PCR conditions were as described above except for the number of the cycles, which was increased to 40. As a reference gene to control the variation in mRNA concentration, zebrafish b-actin1 (drbactin1) was used, employing PCR conditions of 22 cycles at $95^{\circ} \mathrm{C}$ for $30 \mathrm{~s}, 55^{\circ} \mathrm{C}$ for 1 min, and $72^{\circ} \mathrm{C}$ for $1 \mathrm{~min}$. Amplification of genomic
DNA (500 ng), purified from liver, was used as a positive control using the same PCR conditions but with an elongation step of 1 min per $\mathrm{kb}$. An aliquot of the PCR reactions was electrophoresed on $1 \%$ agarose gels containing ethidium bromide and the products were visualized and photographed.

\section{Phylogenetic analysis}

Orthologs of the aquaporin superfamily were obtained from public GenBanks via entrez and BLAST and identified via BLASTP, BLASTN or BLAT from ensembl [32] and the ghost shark (Callorhinchus milii) [81] genome databases. Construction of aquaporin amino acid multiple sequence alignments was achieved using the $\mathrm{t}$ coffee v7.54 suite of tools [82] and ClustalX [83]. Each amino acid alignment was converted to a codon alignment (nucleotide triplets) as described previously [20] and manually adjusted to correct errors using MacVector (MacVector Inc, Cambridge, UK). Three-dimensional alignments of each zebrafish amino acid sequence against crystallographically resolved aquaporin molecules (Additional file 5) were used to identify conserved secondary structures in order to minimize gaps in the $\alpha$ helical regions. Three-dimensional protein wraps were rendered using Cn3D [37] as described previously [24]. Identity and similarity matrices were calculated using MacVector.

Preliminary Bayesian analyses (Mr Bayes v3.1.2; [84]) were run for all sequences, and identical predictions pruned from the alignments. Where possible GenBank sequences were run with predicted variants to validate the latter. Prior to phylogenetic analyses, the alignments were trimmed to remove unrelated $\mathrm{N}$ - and $\mathrm{C}$-terminal regions. To determine the influence of lesser-conserved regions, large gap regions were removed and these alignments tested via Bayesian analyses. This resulted in shorter branch lengths, and an increased incidence of polytomies, but no significant change to the tree topologies.

Each data set was modeled primarily via Bayesian, but also via maximum likelihood or maximum parsimony methods of phylogenetic inference as described previously [20]. Neighbor joining (NJ) methods were used for efficient identification of orthologs. Tree topologies were accepted after validating convergence using Tracer [85] and when the codon and protein tree topologies were congruent. For Bayesian analyses the following settings were used for codon alignments: nucmodel $=4$ by 4, nst $=2$, rates = gamma; and amino acid alignments: aamodel $=$ mixed, with 1,000,000 generations, sampled every 100 generations using 4 chains and a burnin of 3,500. For each run, a majority rule consensus tree together with posterior probabilities from the last 6,500 trees, representing 650,000 generations was arranged using Archeopteryx [86] 
and subsequently rendered with Geneious Pro (Biomatters Ltd, Auckland, New Zealand). Maximum likelihood codon trees were attained using PAUP v4b10 (Sinauer Associates Inc.) and rendered using FigTree v2.2 [87]. Final trees were annotated with accession numbers using Adobe Photoshop.

\section{Sequence accession numbers}

The zebrafish aquaporin cDNA nucleotide sequences reported in this study, which encoded functional proteins when expressed in X. laevis oocytes, have been submitted to the DDBJ/EMBL/GenBank database under the following accession numbers: draqp0a (FJ666326), draqp0b (FJ655389), draqp3a (EU341833), draqp3b (EU341832), draqp4 (FJ666327), draqp7 (FJ655385), draqp8aa (FJ655386), draqp8ab (EU341834), draqp8b (FJ695516), draqp9a (FJ655387), draqp9b (FJ655387), draqp10a (FJ655388), and draqp10b (EU341836).

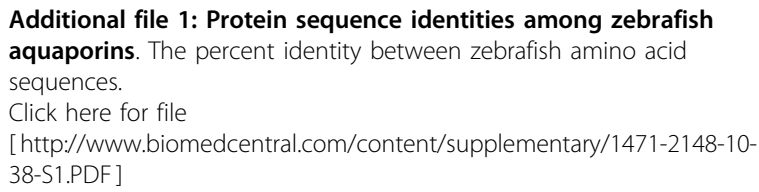

Additional file 4: Phylogenetic analysis of draqp5/1. Maximum likelihood codon trees of zebrafish drapp5/1 (ENSDARG00000038202) exons compared to tetrapod orthologs. (a) Exons 4-8; (b) Exons 1-3. Scale bars indicate nucleotide substitution rate.

Click here for file

[http://www.biomedcentral.com/content/supplementary/1471-2148-1038-S4.PDF ]

\section{Additional file 5: PDB structures used in the study.}

Crystalographically resolved aquaporin molecules used to optimize the amino acid alignments or identify secondary structures.

Click here for file

[http://www.biomedcentral.com/content/supplementary/1471-2148-1038-S5.PDF ]

Additional file 6: Oligonucleotide primers used for RT-PCR analysis Nucleic acid sequences for primers specific for each zebrafish aquaporin mRNA, bactin 1, and expected product size.

Click here for file

[http://www.biomedcentral.com/content/supplementary/1471-2148-1038-S6.PDF ]

\section{Abbreviations}

AQP: aquaporin; BAC: bacterial artificial chromosome; CRNA: capped RNA: EST: expressed sequence tag; Glp: aquaglyceroporin; LG: linkage group; MIP major intrinsic protein; NIP: nodulin 26-like intrinsic protein; NJ: Neighbor joining; NPA: aspargine-proline-alanine; $P_{f}$ : osmotic water permeability; PIP: plasma membrane intrinsic protein; TIP: tonoplast intrinsic proteins; DIP: small and basic intrinsic protein; WGD: whole genome duplication.

\section{Acknowledgements}

This work was supported by the Spanish Ministry of Education and Science AGL2007-60262-AQUACYTE (to JC), by European Commission MRTN-CT-2006035995-Aquaglyceroporins (to JC), and by the Research Council of Norway project \#178837/40 (to RNF). FC was supported by a postdoctoral fellowship from Juan de la Cierva Programme (Spanish Ministry of Education and Science).

\section{Author details}

'Laboratory of Institut de Recerca i Tecnologia Agroalimentàries (IRTA)Institut de Ciències del Mar, Consejo Superior de Investigaciones Científicas (CSIC), 08003 Barcelona, Spain. Génomique et Physiologie des Poissons, Université Bordeaux 1, UMR NuAGe, 33405 Talence, France. ${ }^{3}$ Center for Protein Engineering, University of Liège, B-4000 Liège, Belgium. ${ }^{4}$ Department of Biology, University of Bergen, Bergen High Technology Center, N-5020 Bergen, Norway. ${ }^{5}$ Centro de Investigación Biomédica en Red de Enfermedades Hepáticas y Digestivas (CIBERehd), 08036 Barcelona, Spain.

\section{Authors' contributions}

ATS, MC and FC carried out the cloning, functional analysis in oocytes and gene expression experiments. RNF, JL and JC analyzed the data and performed structural analyses, and RNF conducted the phylogenetic analyses. JC conceived and coordinated the study, participated in the design of the experiments, and together with RNF wrote the final version of the manuscript. All authors read and approved the final manuscript.

Received: 11 November 2009

Accepted: 11 February 2010 Published: 11 February 2010

\section{References}

1. King LS, Kozono D, Agre P: From structure to disease: the evolving tale of aquaporin biology. Nat Rev Mol Cell Biol 2004, 5:687-698.

2. Zardoya R: Phylogeny and evolution of the major intrinsic protein family. Biol Cell 2005, 97:397-414

3. Preston GM, Caroll TP, Guggino WB, Agre P: Appearance of water channels in Xenopus oocytes expressing red-cell CHIP28 protein. Science 1992, 256:385-387

4. Quigley F, Rosenberg JM, Shachar-Hill Y, Bohnert HJ: From genome to function: the Arabidopsis aquaporins. Genome Biol 2001, 3: research0001.1-0001.17.

5. Maurel C, Javot H, Lauvergeat V, Gerbeau P, Tournaire C, Santoni V, Heyes J: Molecular physiology of aquaporins in plants. Int Rev Cytol 2002, 215:105-48

6. Zardoya R, Ding X, Kitagawa $Y$, Chrispeels MJ: Origin of plant glycerol transporters by horizontal gene transfer and functional recruitment. Proc Natl Acad Sci USA 2002, 99:14893-14896.

7. Smith BL, Agre P: Erythrocyte Mr 28,000 transmembrane protein exists as a multisubunit oligomer similar to channel proteins. J Biol Chem 1991, 266:6407-6415

8. Verbavatz JM, Brown D, Sabolic I, Valenti G, Ausiello DA, Van Hoek AN, Ma T, Verkman AS: Tetrameric assembly of CHIP28 water channels in liposomes and cell membranes: a freeze-fracture study. J Cell Biol 1993, 123:605-618

9. Walz T, Hirai T, Murata K, Heymann JB, Mitsuoka K, Fujiyoshi Y, Smith BL Agre $P$, Engel A: The three-dimensional structure of aquaporin-1. Nature 1997, 387:624-627.

10. Hoek AN, Hom ML, Luthjens LH, de Jong MD, Dempster JA, van Os CH: Functional unit of $30 \mathrm{kDa}$ for proximal tubule water channels as revealed by radiation inactivation. J Biol Chem 1991, 266:16633-16635.

11. Preston GM, Jung JS, Guggino WB, Agre P: The mercury-sensitive residue at cysteine 189 in the CHIP28 water channel. J Biol Chem 1993, 268:17-20. 
12. Martinez AS, Cutler CP, Wilson GD, Phillips C, Hazon N, Cramb G: Regulation of expression of two aquaporin homologs in the intestine of the European eel: effects of seawater acclimation and cortisol treatment. Am J Physiol Regul Integr Comp Physiol 2005, 288:R1733-R1743.

13. Watanabe S, Kaneko T, Aida K: Aquaporin-3 expressed in the basolateral membrane of gill chloride cells in Mozambique tilapia Oreochromis mossambicus adapted to freshwater and seawater. J Exp Biol 2005, 208:2673-2682

14. Cutler CP, Martinez AS, Cramb G: The role of aquaporin 3 in teleost fish. Comp Biochem Physiol 2007, 148A:82-91.

15. Giffard-Mena I, Boulo V, Aujoulat F, Fowden H, Castille R, Charmantier G, Cramb G: Aquaporin molecular characterization in the sea-bass (Dicentrachus labrax): the effect of salinity on AQP1 and AQP3 expression. Comp Biochem Physiol 2007, 148A:430-444.

16. Raldúa D, Otero D, Fabra M, Cerdà J: Differential localization and regulation of two aquaporin-1 homologs in the intestinal epithelia of the marine teleost Sparus aurata. Am J Physiol Regul Integr Comp Physiol 2008, 294:R993-R1003.

17. Fabra M, Raldúa D, Power DM, Deen PM, Cerdà J: Marine fish egg hydration is aquaporin-mediated. Science 2005, 307:545

18. Fabra M, Raldúa D, Bozzo MG, Deen PM, Lubzens E, Cerdà J: Yolk proteolysis and aquaporin-10 play essential roles to regulate fish oocyte hydration during meiosis resumption. Dev Biol 2006, 295:250-262.

19. Finn RN: The maturational disassembly and differential proteolysis of paralogous vitellogenins in a marine pelagophil teleost: a conserved mechanism of oocyte hydration. Biol Reprod 2007, 76:936-948.

20. Finn RN, Kristoffersen BA: Vertebrate vitellogenin gene duplication in relation to the " $3 R$ hypothesis": correlation to the pelagic egg and the oceanic radiation of teleosts. PLOS ONE 2007, 2:e169.

21. Kolarevic J, Nerland A, Nilsen F, Finn RN: Goldsinny wrasse (Ctenolabrus rupestris) is an extreme vtgAa-type pelagophil teleost. Mol Reprod Dev 2008, 75:1011-1020.

22. Tingaud-Sequeira A, Chauvigné F, Fabra M, Lozano J, Raldúa D, Cerdà J: Structural and functional divergence of two fish aquaporin-1 water channels following teleost-specific gene duplication. BMC Evol Biol 2008 8:259.

23. Finn RN, Kolarevic J, Kongshaug H, Nilsen F: Evolution and differential expression of a vertebrate vitellogenin gene cluster. BMC Evol Biol 2009, 9:2.

24. Kristoffersen BA, Nerland A, Nilsen F, Kolarevic J, Finn RN: Genomic and proteomic analyses reveal non-neofunctionalized vitellogenins in a basal clupeocephalan, the Atlantic herring, and point to the origin of maturational yolk proteolysis in marine teleosts. Mol Biol Evol 2009, 26:1029-1044.

25. Postlethwait JH: The zebrafish genome: a review and msx gene case study. Genome Dyn 2006, 2:183-197.

26. Hsu CH, Wen ZH, Lin CS, Chakraborty C: The zebrafish model: use in studying cellular mechanisms for a spectrum of clinical disease entities. Curr Neur Res 2007, 4:111-120.

27. Wingert RA, Davidson AJ: The zebrafish pronephros: A model to study nephron segmentation. Kidney Int 2008, 73:1120-1127.

28. Verkman AS: Mammalian aquaporins: diverse physiological roles and potential clinical significance. Exp Rev Mol Med 2008, 10:e13.

29. Wain HM, Bruford EA, Lovering RC, Lush MJ, Wright MW, Povey S: Guidelines for human gene nomenclature. Genomics 2002, 79:464-470.

30. The Zebrafish Information Network. http://zfin.org.

31. Zardoya R, Villalba S: A phylogenetic framework for the aquaporin family in eukaryotes. J Mol Evol 2001, 52:391-404.

32. Ensembl Genome Browser. http://www.ensemblorg

33. Amores A, Force A, Yan YL, Joly L, Amemiya C, Fritz A, Ho RK, Langeland J, Prince V, Wang YL, Westerfield M, Ekker M, Postlethwait JH: Zebrafish hox clusters and vertebrate genome evolution. Science 1998, 282:1711-1714.

34. Christoffels A, Koh EG, Chia JM, Brenner S, Aparicio S, Venkatesh B: Fugu genome analysis provides evidence for a whole-genome duplication early during the evolution of ray-finned fishes. Mol Biol Evol 2004, 21:1146-1151

35. Jaillon O, Aury JM, Brunet F, Petit JL, Stange-Thomann N, Mauceli E, Bouneau L, Fischer C, Ozouf-Costaz C, Bernot A, Nicaud S, Jaffe D, Fisher S, Lutfalla G, Dossat C, Segurens B, Dasilva C, Salanoubat M, Levy M, Boudet N, Castellano S, Anthouard V, Jubin C, Castelli V, Katinka M, Vacherie B, Biemont C, Skalli Z, Cattolico L, Poulain J, et al: Genome duplication in the teleost fish Tetraodon nigroviridis reveals the early vertebrate protokaryotype. Nature 2004, 431:946-957.

36. Crow KD, Stadler PF, Lynch VJ, Amemiya C, Wagner GP: The "fish-specific" Hox cluster duplication is coincident with the origin of teleosts. Mol Biol Evol 2006, 23:121-136.

37. National Center for Biotechnology Information (NCBI). http://blast.ncbi. nlm.nih.gov.

38. Kasahara M, Naruse K, Sasaki S, Nakatani Y, Qu W, et al: The medaka draft genome and insights into vertebrate genome evolution. Nature 2007. 447:714-719.

39. Froger A, Tallur B, Thomas D, Delamarche C: Prediction of functional residues in water channels and related proteins. Protein Sci 2000, 7:1458-1468.

40. Cheng A, van Hoek AN, Yeager M, Verkman AS, Mitra AK: Threedimensional organization of a human water channel. Nature 1997, 387:627-630.

41. Murata K, Mitsuoka K, Hirai T, Walz T, Agre P, Heymann JB, Engel A, Fujiyoshi Y: Structural determinants of water permeation through aquaporin-1. Nature 2000, 407:599-605.

42. Sui $H$, Han BG, Lee JK, Walian $P$, Jap BK: Structural basis of water specific transport through the AQP1 water channel. Nature 2001, 414:872-878.

43. Beitz E, Wu B, Holm LM, Schultz JE, Zeuthen T: Point mutations in the aromatic/arginine region in aquaporin 1 allow passage of urea, glycerol, ammonia, and protons. Proc Natl Acad Sci USA 2006, 103:269-274.

44. de Groot BL, Frigato T, Helms V, Grubmüller H: The mechanism of proton exclusion in the aquaporin-1 water channel. J Mol Biol 2003, 333:279-293.

45. Ishibashi K: Aquaporin subfamily with unusual NPA boxes. Biochim Biophys Acta 2006, 1758:989-993.

46. de Groot BL, Grubmüller H: Water permeation across biological membranes: mechanism and dynamics of aquaporin-1 and GlpF. Science 2001, 294:2353-2357.

47. de Groot BL, Grubmüller H: The dynamics and energetics of water permeation and proton exclusion in aquaporins. Curr Opin Struct Biol 2005, 15:176-183.

48. Hub JS, de Groot BL: Mechanism of selectivity in aquaporins and aquaglyceroporins. Proc Natl Acad Sci USA 2008, 105:1198-1203.

49. Verkman AS, Ives HE: Water permeability and fluidity of renal basolateral membranes. Am J Physiol 1986, 250:F633-F643.

50. Calamita G, Bishai WR, Preston GM, Guggino WB, Agre P: Molecular cloning and characterization of AqpZ, a water channel from Escherichia coli.. J Biol Chem 1995, 270:29063-29066.

51. Zeuthen T, Klaerke DA: Transport of water and glycerol in aquaporin 3 is gated by $\mathrm{H}^{+}$. J Biol Chem 1999, 274:21631-21636.

52. Maclver B, Cutler CP, Yin J, Hill MG, Zeidel ML, Hill WG: Expression and functional characterization of four aquaporin water channels from the European eel (Anguilla anguilla). J Exp Biol 2009, 212:2856-2863.

53. Ishibashi K, Sasaki S, Fushimi K, Uchida S, Kuwahara M, Saito H, Furukawa T, Nakajima K, Yamaguchi Y, Gojobori T, Marumo : Molecular cloning and expression of a member of the aquaporin family with permeability to glycerol and urea in addition to water expressed at the basolateral membrane of kidney collecting duct cells. Proc Natl Acad Sci USA 1994, 91:6269-6273.

54. Echevarria M, Windhager EE, Tate SS, Frindt G: Cloning and expression of AQP3, a water channel from the medullary collecting duct of rat kidney. Proc Natl Acad Sci USA 1994, 91:10997-1001.

55. Maurel C, Reizer J, Schroeder JI, Chrispeels MJ: The vacuolar membrane protein gamma-TIP creates water specific channels in Xenopus oocytes. EMBO J 1993, 12:2241-2247.

56. Daniels MJ, Chaumont F, Mirkov TE, Chrispeels MJ: Characterization of a new vacuolar membrane aquaporin sensitive to mercury at a unique site. Plant Cell 1996, 8:587-599.

57. Itoh T, Rai T, Kuwahara M, Ko SB, Uchida S, Sasaki S, Ishibashi K: Identification of a novel aquaporin, AQP12, expressed in pancreatic acinar cells. Biochem Biophys Res Commun 2005, 330:832-838.

58. Gorelick DA, Praetorius J, Tsunenari T, Nielsen S, Agre P: Aquaporin-11: a channel protein lacking apparent transport function expressed in brain. BMC Biochem 2006, 7:14.

59. Morishita Y, Matsuzaki T, Hara-chikuma M, Andoo A, Shimono M, Matsuki A, Kobayashi K, lkeda M, Yamamoto T, Verkman A, Kusano E, Ookawara S, Takata K, Sasaki S, Ishibashi K: Disruption of aquaporin-11 produces 
polycystic kidneys following vacuolization of the proximal tubule. Mol Cell Biol 2005, 25:7770-7779.

60. Yakata K, Hiroaki Y, Ishibashi K, Sohara E, Sasaki S, Mitsuoka K, Fujiyoshi Y: Aquaporin-11 containing a divergent NPA motif has normal water channel activity. Biochim Biophys Acta 2007, 1768:688-693.

61. Mobasheri A, Marples D: Expression of the AQP-1 water channel in normal human tissues: a semiquantitative study using tissue microarray technology. Am J Physiol Cell Physiol 2004, 286:C529-C537.

62. Aoki M, Kaneko T, Katoh F, Hasegawa S, Tsutsui N, Aida K: Intestinal water absorption through aquaporin 1 expressed in the apical membrane of mucosal epithelial cells in seawater-adapted Japanese eel. J Exp Biol 2003, 206:3495-3505.

63. Tenckhoff S, Hollborn M, Kohen L, Wolf S, Wiedemann P, Bringmann A: Diversity of aquaporin mRNA expressed by rat and human retinas. Neuroreport 2005, 16:53-56.

64. Tchekneva EE, Khuchua Z, Davis LS, Kadkina V, Dunn SR, Bachman S, Ishibashi K, Rinchik EM, Harris RC, Dikov MM, Breyer MD: Single amino acid substitution in aquaporin 11 causes renal failure. J Am Soc Nephrol 2008, 19:1955-1964

65. Virkki LV, Cooper GJ, Boron WF: Cloning and functional expression of an MIP (AQPO) homolog from killifish (Fundulus heteroclitus) lens. Am J Physiol Regul Integr Comp Physiol 2001, 281:R1994-R2003.

66. Tingaud-Sequeira A, Zapater C, Chauvigné F, Otero D, Cerdà J: Adaptive plasticity of killifish (Fundulus heteroclitus) embryos: dehydrationstimulated development and differential aquaporin-3 expression. Am J Physiol Regul Integr Comp Physiol 2009, 296:R1041-R1052.

67. Broekhuyse RM, Kuhlmann ED, Stols AL: Lens membranes. II. Isolation and characterization of the main intrinsic polypeptide (MIP) of bovine lens fiber membranes. Exp Eye Res 1976, 23:365-371.

68. McConnell NA, Yunus RS, Gross SA, Bost KL, Clemens MG, Hughes FM Jr: Water permeability of an ovarian antral follicle is predominantly transcellular and mediated by aquaporins. Endocrinology 2002, 143:2905-2912.

69. Rojek A, Praetorius J, Frøkiaer J, Nielsen S, Fenton RA: A current view of the mammalian aquaglyceroporins. Annu Rev Physiol 2008, 70:301-27.

70. Santos CR, Estêvão MD, Fuentes J, Cardoso JC, Fabra M, Passos AL, Detmers FJ, Deen PM, Cerdà J, Power DM: Isolation of a novel aquaglyceroporin from a marine teleost (Sparus auratus): function and tissue distribution. J Exp Biol 2004, 207:1217-1227.

71. Morinaga T, Nakakoshi M, Hirao A, Imai M, Ishibashi K: Mouse aquaporin 10 gene (AQP10) is a pseudogene. Biochem Biophys Res Commun 2002, 294:630-634

72. Hibuse T, Maeda N, Funahashi T, Yamamoto K, Nagasawa A, Mizunoya W, Kishida K, Inoue K, Kuriyama H, Nakamura T, Fushiki T, Kihara S, Shimomura I: Aquaporin 7 deficiency is associated with development of obesity through activation of adipose glycerol kinase. Proc Natl Acad Sci USA 2005, 102:10993-10998.

73. Hara-Chikuma M, Sohara E, Rai T, Ikawa M, Okabe M, Sasaki S, Uchida S, Verkman AS: Progressive adipocyte hypertrophy in aquaporin-7-deficient mice: adipocyte glycerol permeability as a novel regulator of fat accumulation. J Biol Chem 2005, 280:15493-15496.

74. Wu B, Beitz E: Aquaporins with selectivity for unconventional permeants. Cell Mol Life Sci 2007, 64:2413-21.

75. Westerfield M: The zebrafish book. Seattle, Oregon, University of Oregon Press, 21995.

76. Altschul SF, Madden TL, Schaffer AA, Zhang J, Anang Z, Miller W, Lipman DJ: Gapped BLAST and PSI-BLST: a new generation of protein database search programs. NuCl Acids Res 1997, 25:3389-3402.

77. Hirokawa T, Boon-Chieng S, Mitaku S: SOSUI: classification and secondary structure prediction system for membrane proteins. Bioinformatics 1998, 14:378-379.

78. Krogh A, Larsson B, von Heijne G, Sonnhammer EL: Predicting transmembrane protein topology with a hidden Markov model: application to complete genomes. J Mol Biol 2001, 305:567-580.

79. Käll L, Krogh A, Sonnhammer EL: Advantages of combined transmembrane topology and signal peptide prediction-the Phobius web server. Nucleic Acids Res 2007, 35:W429-W432.

80. Deen PMT, Verdijk MAJ, Knoers $N$, Wieringa B, Monnens $L A$, van Os $C H$, van Oost BA: Requirement of human renal water channel aquaporin-2 for vasopressin-dependent concentration of urine. Science 1994, 264:92-95.

81. Elephant Shark Genome Project. http://esharkgenome.imcb.a-star.edu.sg.
82. Notredame C, Higgins DG, Herringa J: T-Coffee: A novel method for fast and accurate multiple sequence alignment. J Mol Biol 2000, 302:205-217.

83. Chenna R, Sugawara H, Koike T, Lopez R, Gibson TJ, Higgins DG, Thompson JD: Multiple sequence alignment with the Clustal series of programs. Nucleic Acids Res 2003, 31:3497-3500.

84. Ronquist F, Huelsenbeck JP: MrBayes 3: Bayesian phylogenetic inference under mixed models. Bioinformatics 2003, 19:1572-1574.

85. Rambaut A, Drummond AJ: Tracer v1.4. 2007http://beast.bio.ed.ac.uk/Tracer.

86. Zmasek CM, Eddy SR: ATV: display and manipulation of annotated phylogenetic trees. Bioinformatics 2001, 17:383-384.

87. Rambaut A: FigTree.http://tree.bio.ed.ac.uk/software.

doi:10.1186/1471-2148-10-38

Cite this article as: Tingaud-Sequeira et al:: The zebrafish genome encodes the largest vertebrate repertoire of functional aquaporins with dual paralogy and substrate specificities similar to mammals. $B M C$ Evolutionary Biology 2010 10:38.

\section{Submit your next manuscript to BioMed Central and take full advantage of:}

- Convenient online submission

- Thorough peer review

- No space constraints or color figure charges

- Immediate publication on acceptance

- Inclusion in PubMed, CAS, Scopus and Google Scholar

- Research which is freely available for redistribution
C Biomed Central 